\title{
Triamterene in the Treatment of Hypertension: More Than Just Potassium Sparing?
}

\author{
Gerald W. Smetana, M.D.
}

Division of General Medicine and Primary Care, Beth Israel Deaconess Medical Center, Harvard Medical School, Boston, MA, USA.

J Gen Intern Med 31(1):7-8

DOI: $10.1007 / \mathrm{s} 11606-015-3515-Z$

(c) Society of General Internal Medicine 2015

$\mathrm{H}$ ypertension is the most commonly encountered chronic medical condition in primary care practice and is a major risk factor for stroke and coronary artery disease. For decades, thiazide diuretics have been a recommended first line option for antihypertensive therapy. The Seventh Report of the Joint National Committee on Prevention, Detection, Evaluation, and Treatment of High Blood Pressure (JNC 7) recommended thiazides as first line therapy unless a compelling indication existed for another agent. JNC-8 recommends diuretics as one of four acceptable first line options for non-black patients without chronic kidney disease (other options include angiotensin-converting enzyme (ACE) inhibitors, angiotensin receptor blockers, or calcium channel blockers), and as one of two acceptable options for African American patients without chronic kidney disease (the other option is calcium channel blockers). ${ }^{1}$

Hypokalemia frequently results from the administration of thiazide diuretics. This effect is more common with chlorthalidone than hydrochlorothiazide (HCTZ) or indapamide, and occurs in a dose dependent fashion. In the Antihypertensive and Lipid-Lowering Treatment to Prevent Heart Attack Trial (ALLHAT), one of the largest trials of antihypertensive therapy $(n=33,357)$, mean potassium levels at 4 years fell $0.2 \mathrm{mEq} / \mathrm{L}$ among chlorthalidone treated patients, and potassium fell below $3.5 \mathrm{mEq} / \mathrm{L}$ in $8.5 \%$ of patients at 4 years (as opposed to $1.9 \%$ of amlodipine treated patients). ${ }^{2}$ Hypokalemia, when it occurs, usually appears within the first 2 weeks after initiating diuretic therapy. If hypokalemia occurs, clinicians have the option of beginning potassium chloride replacement therapy (typical doses are $20-40 \mathrm{mEq}$ per day for patients with normal renal function), or beginning a potassium sparing diuretic. Potassium sparing diuretics include triamterene and amiloride (epithelial sodium channel inhibitors) and spironolactone and eplerenone (mineralocorticoid receptor antagonists). The choice of potassium replacement or a potassium-sparing diuretic has been typically left to the discretion of the clinician, as no evidence has suggested that the addition of potassium-sparing diuretics provides

Published online September 18, 2015 additional value in terms of blood pressure lowering or a reduction in cardiovascular events. From the patient perspective, a disadvantage of potassium replacement is the requirement to add either large pills that are often difficult to swallow, or a salty tasting solution to the thiazide.

No studies exist of triamterene as monotherapy for the treatment of hypertension. In a recent Cochrane systematic review, authors evaluated the blood pressure lowering effect of potassium-sparing diuretics that block the epithelial sodium channel when given in combination with another antihypertensive agent. ${ }^{3}$ Only six trials of 496 patients existed; all six studies were performed in the 1980s. Two trials $(n=211)$ evaluated the incremental benefit of triamterene $50 \mathrm{mg}$ per day when added to chlorthalidone at 25 to $50 \mathrm{mg}$ per day. The addition of triamterene provided no incremental reduction in systolic blood pressure $(-0.01,95 \% \mathrm{CI}-3.63$ to 3.61$)$, or diastolic blood pressure $(+0.20,95 \% \mathrm{CI}-2.01$ to 2.41$)$, but total sample sizes were too small to draw any meaningful conclusions.

In this issue of JGIM, Tu and colleagues queried a large network electronic medical record system to determine the incremental blood pressure lowering effect of triamterene. ${ }^{4}$ They identified 17,291 patients with a diagnosis of hypertension over an 8-year period and divided these patients into those with and without a pharmacy claim for triamterene. Patients who received triamterene were more likely to be female or African American, and less likely to have diabetes, coronary artery disease, congestive heart failure, a history of stroke, or chronic obstructive pulmonary disease. A direct comparison of the blood pressure values in these two groups would be confounded by substantial selection bias due to the nonrandom assignment between the groups.

$\mathrm{Tu}$ et al. used a novel approach to attempt to correct for limitations inherent in these observational data. They used propensity score matching to estimate the probability that a patient would receive a particular treatment, based on logistic regression that adjusted for 14 clinical characteristics. They then stratified patients into quartiles of estimated propensity and compared the recorded blood pressures for those who had or had not received triamterene. They evaluated separately the impact of adding triamterene to HCTZ or to combinations of drugs that included HCTZ. They present no data on chlorthalidone use; in their network, triamterene was most commonly prescribed as a fixed combination pill with HCTZ (initial dose was HCTZ $25 \mathrm{mg}$ daily and triamterene $37.5 \mathrm{mg}$ daily). 
After propensity score matching, patients who received HCTZ and triamterene had systolic blood pressures that were $3.8 \mathrm{mg} \mathrm{Hg}$ lower (SD 0.70, $p<0.0001$ ) than those who received HCTZ alone. There was a nonsignificant trend towards lower diastolic blood pressures with combination therapy (0.90 mm Hg, SD 0.55, $p=0.1029$ ). The results were similar when comparisons were made for patients on combination therapies that included ACE inhibitors, calcium channel blockers, and beta blockers. The range of systolic blood pressure reductions was 1.84 to 3.80 (all comparisons were significant). Potassium levels were identical $(4.2 \mathrm{mEq})$ in both the triamterene and non-triamterene groups. The authors did not provide data on cardiovascular outcomes or mortality.

This is the first large study to suggest that triamterene, in addition to its potassium sparing capabilities, lowers blood pressure. This is a well-designed and commendable study that was ambitious in scope. An important potential limitation of the current study is the potential for residual confounding, despite propensity score matching, as noted by the authors. The degree of blood pressure reduction attributed to triamterene was modest at $3 \mathrm{~mm} \mathrm{Hg}$ systolic; there was no significant diastolic blood pressure reduction. The authors comment that this is comparable to that seen with other first line drugs. However, the studies they cite included patients without hypertension and were primarily of fixed dose antihypertensive agents. ${ }^{5,6}$ In ALLHAT, which more closely reflected actual clinical practice, blood pressure reductions with antihypertensive therapy averaged 10-12 mg $\mathrm{Hg}$ systolic and $9 \mathrm{~mm} \mathrm{Hg}$ diastolic. ${ }^{2}$ While a small number of patients in ALLHAT received more than one drug during the duration of the study, this represented only $5-13 \%$ of subjects.

Triamterene is not without risk. It commonly causes crystalluria, can cause hyperuricemia and hyperkalemia, and rarely can cause triamterene renal stones or acute kidney injury. ${ }^{7}$ The risk of acute kidney injury is increased with concurrent therapy with nonsteroidal anti-inflammatory drugs (NSAIDs). An advantage is the potential for increased adherence due to a single tablet formulation that obviates the need for a separate potassium supplement. Serum potassium levels, in the current study, did not differ between the two groups. The non-triamterene group would include patients whose serum potassium did not fall substantially after initiating HCTZ, and those in whom hypokalemia developed and was treated with potassium supplementation. It is not known what percent of the non-triamterene treated patients required potassium supplementation, but the current study suggests that potassium repletion was adequate when required and was not a barrier to the safe use of HCTZ.

Over the past decade, a growing literature has emerged which suggests that not all antihypertensive agents reduce cardiovascular disease to the same extent. ${ }^{8}$ For example, despite comparable blood pressure reduction, alpha-blockers ${ }^{2}$ and betablockers ${ }^{9}$ do not reduce cardiovascular risk to the same extent as other antihypertensive agents. As such, they are no longer recommended as initial treatment for hypertension. ${ }^{1}$ Therefore, it is not appropriate to extrapolate a proxy endpoint of blood pressure reduction to a clinical endpoint of cardiovascular events. While plausible, it is unknown whether the $3 \mathrm{~mm} \mathrm{Hg}$ systolic blood pressure reduction attributed to triamterene, in the current study, would lead to a reduction in cardiovascular events. A single randomized trial of triamterene in combination with HCTZ that evaluated cardiovascular outcomes demonstrated a reduction in cardiovascular, but not total, mortality. ${ }^{10}$

I applaud this study by $\mathrm{Tu}$ and colleagues for the novel approach to an important clinical question. They have shown that triamterene most likely reduces blood pressure modestly when given in combination with HCTZ alone, or in combination with other antihypertensive agents. The effect on cardiovascular outcomes is unknown. Given the potential for improved adherence, and the modest blood pressure effect, the addition of triamterene remains an acceptable alternative to potassium replacement for patients who develop hypokalemia while taking thiazide diuretics for the treatment of hypertension. The available evidence is not sufficient to recommend triamterene as the preferred strategy for thiazide induced hypokalemia.

Conflict of Interest: Dr. Smetana declares that he does not have a financial conflict of interest.

Corresponding Author: Gerald W. Smetana, M.D.; Division of General Medicine and Primary Care, Beth Israel Deaconess Medical Center Harvard Medical School, 330 Brookline Ave., Boston MA 02215, USA (e-mail: gsmetana@bidmc.harvard.edu).

\section{REFERENCES}

1. James PA, Oparil S, Carter BL, et al. 2014 evidence-based guideline for the management of high blood pressure in adults: report from the panel members appointed to the Eighth Joint National Committee (JNC 8). JAMA. 2014;311(5):507-20.

2. Officers A, Coordinators for the ACRGTA, Lipid-Lowering Treatment to Prevent Heart Attack T. Major outcomes in high-risk hypertensive patients randomized to angiotensin-converting enzyme inhibitor or calcium channel blocker vs diuretic: the Antihypertensive and Lipid-Lowering Treatment to Prevent Heart Attack Trial (ALLHAT). JAMA. 2002;288(23):2981-97.

3. Heran BS, Chen JM, Wang JJ, Wright JM. Blood pressure lowering efficacy of potassium-sparing diuretics (that block the epithelial sodium channel) for primary hypertension. Cochrane Database Syst Rev. 2012;11:CD008167.

4. Tu W, Decker BS, He Z, et al. Triamterene enhances the blood pressure lowering effect of hydrochlorothiazide in patients with hypertension. J Gen Intern Med. 2015. doi:10.1007/s11606-015-3469-1.

5. Yusuf S, Sleight P, Pogue J, Bosch J, Davies R, Dagenais G. Effects of an angiotensin-converting-enzyme inhibitor, ramipril, on cardiovascular events in high-risk patients. The Heart Outcomes Prevention Evaluation Study Investigators. N Engl J Med. 2000;342(3):145-53.

6. Pitt B, Byington RP, Furberg CD, et al. Effect of amlodipine on the progression of atherosclerosis and the occurrence of clinical events. PREVENT Investigators. Circulation. 2000;102(13):1503-10.

7. Perazella MA. Crystal-induced acute renal failure. Am J Med. 1999; 106(4):459-65.

8. Smetana GW. Newer is not always better: all antihypertensive medications do not equally reduce cardiovascular risk. J Gen Intern Med. 2012;27(6):618-20.

9. Wiysonge CS, Bradley HA, Volmink J, Mayosi BM, Mbewu A, Opie LH. Beta-blockers for hypertension. Cochrane Database Syst Rev. 2012;8:CD002003.

10. Amery A, Birkenhager W, Brixko $\mathbf{P}$, et al. Mortality and morbidity results from the European Working Party on High Blood Pressure in the Elderly trial. Lancet. 1985;1(8442):1349-54. 